\title{
CORRECTION
}

\section{Diabetic kidney disease}

Merlin C. Thomas, Michael Brownlee, Katalin Susztak, Kumar Sharma, Karin A. M. Jandeleit-Dahm, Sophia Zoungas, Peter Rossing, Per-Henrik Groop and Mark E. Cooper

Nat. Rev. Dis. Primers article number: 15018; doi:10.1038/nrdp.2015.18; published online 30 July 2015

In the version of the article originally published, Figure 1 incorrectly stated that the DEMAND study with 2,862 participants was conducted in an Indigenous Australian population. This analysis was conducted using participants from Asia. The article has now been corrected. 